\title{
Alignment of liquid crystals with periodic submicron structures ablated in polymeric and indium tin oxide surfaces
}

\author{
M. Behdani \\ Research Institute for Materials, University of Nijmegen, Toernooiveld 1-6525 ED Nijmegen, The \\ Netherlands, Microelectronic Research Laboratory, University of Mashhad, Mashhad, Iran, and Department \\ of Physics, University of Birjand, Birjand, Iran
}

S. H. Keshmiri

Microelectronic Research Laboratory, University of Mashhad, Mashhad, Iran

S. Soria, ${ }^{a)}$ M. A. Bader, J. Ihlemann, and G. Marowsky

Laser Laboratorium Goettingen, Hans Adolf Krebs Weg 1, D-37077 Goettingen, Germany

Th. Rasing

Research Institute for Materials, University of Nijmegen, Toernooiveld 1-6525 ED Nijmegen, The Netherlands

(Received 5 July 2002; accepted 17 February 2003)

\begin{abstract}
We show that a periodic nanostructure patterned into a polymeric or indium tin oxide (ITO) surface is capable of aligning liquid crystal (LC) molecules. Gratings of different depths were created on thin polymeric or ITO surfaces with submicron and micron periods by superposition of ultraviolet plane waves. The depth of the gratings was varied by changing the fluence of the laser. This method allows to pattern orientations over small areas and does not suffer from the disadvantages of rubbing based alignment methods. LC alignment was tested by forming twisted nematic cells. Anchoring energies were calculated from measurements of the twist angles. (C) 2003 American Institute of Physics. [DOI: 10.1063/1.1565703]
\end{abstract}

Conventionally, molecular alignment is achieved by putting the liquid crystal (LC) in contact with an anisotropic substrate. For commercial devices, the anisotropy is usually obtained by means of unidirectional mechanical rubbing of a polymer layer ${ }^{1}$ coated on a surface. The rubbing induces grooves in the polymer surface and the LC molecules prefer to align parallel to the direction of the grooves. ${ }^{2,3}$ Though a very cheap and reliable method, rubbing has the disadvantage of creating dust and electrostatic charges, which can both be detrimental for the further development of LC devices. Alternative, contact free methods of inducing alignment would therefore be advantageous, such as photopolymers. ${ }^{4}$ Also, future LC devices will rely on patterned orientations over small areas. Such patterning cannot be obtained by the standard rubbing technique but it is possible, for example, using nanopatterning by atomic force microscope $(\mathrm{AFM})^{5}$ on polymeric and even directly on indium tin oxide (ITO) ${ }^{6}$ surfaces though this approach is not contact free. Since a grating can be considered as a fine but contact free rubbing, a similar mechanism could be responsible for the alignment of liquid crystal molecules in contact with a grating.

A submicron patterning of many polymeric materials, e.g., polyimide (PI), polymethylmethacrylate (PMMA), polyethylene terephthalate, can be obtained with nanosecond (ns) pulses. ${ }^{7}$ However, ITO has a higher thermal diffusivity and, as a consequence, the use of femtosecond (fs) pulses for ablation and structuring leads to a better performance compared to ns-pulse ablation. The high coherence of the fs-laser

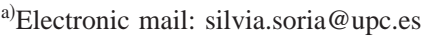

pulses also leads to an improvement of the optical contrast.

In this letter we show how submicron periodic surface structures generated by fs and ns pulse UV laser ablation in PI, PMMA, and ITO. Our method allows to pattern LC orientations over small areas and leads to well oriented LC cells with a strong anchoring energy.

The ablation experiments were performed with a short pulse $\mathrm{KrF}$-laser system ${ }^{8}$ and an ArF-laser system. The output is about $10 \mathrm{~mJ}$ at $248 \mathrm{~nm}$ with pulse duration of $500 \mathrm{fs}$ and about $400 \mathrm{~mJ}$ at $193 \mathrm{~nm}$ with pulse duration of $20 \mathrm{~ns}$, respectively. For mask imaging a Schwarzschild type reflective objective was used. This ensures high power transmission in the UV and prohibits pulse front distortion for femtosecond pulses. The objectives had a numerical aperture of 0.3 and 0.4 , and were used with high demagnification of $15 \times$ and $25 \times$, respectively. The beam $(248 \mathrm{~nm}, 500 \mathrm{fs} ; 193 \mathrm{~nm}, 20$ $\mathrm{ns}$ ) is diffracted from a transmission grating (55 lines $/ \mathrm{mm} \mathrm{Cr}$ on quartz) and then illuminates the sample surface using only the plus and minus first diffraction orders to create a pure sinusoidal intensity modulation on the sample with a period of $\approx 0.4, \approx 0.7$, and $1 \mu \mathrm{m}$. The zeroth order of diffraction was blocked in order to increase the created line density by a factor of 2, for periods of $400 \mathrm{~nm}$. The fluence was varied with a dielectric attenuator (see Table I).

An ITO layer of $19 \mathrm{~nm}$ was coated by rf sputtering on a

TABLE I. Description of the laser systems.

\begin{tabular}{lcccc}
\hline \hline Laser & Characteristics & Fluence $\left(\mathrm{mJ} / \mathrm{cm}^{2}\right)$ & Spot shape & Spot size $(\mu \mathrm{m})$ \\
\hline $\mathrm{KrF}$ & $248 \mathrm{~nm}, 500 \mathrm{fs}$ & $13-230$ & Circular & 60 \\
$\mathrm{ArF}$ & $193 \mathrm{~nm}, 20 \mathrm{~ns}$ & $110-350$ & Square & 90 \\
\hline \hline
\end{tabular}


(a)

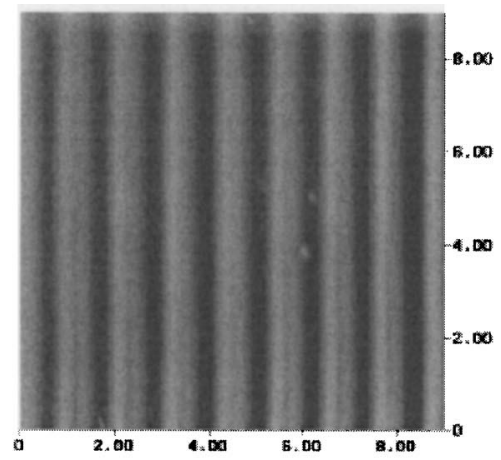

(b)

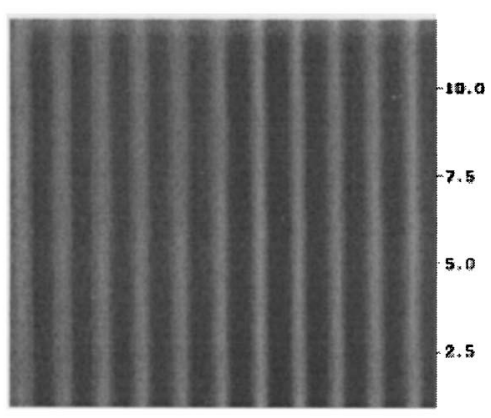

(c)

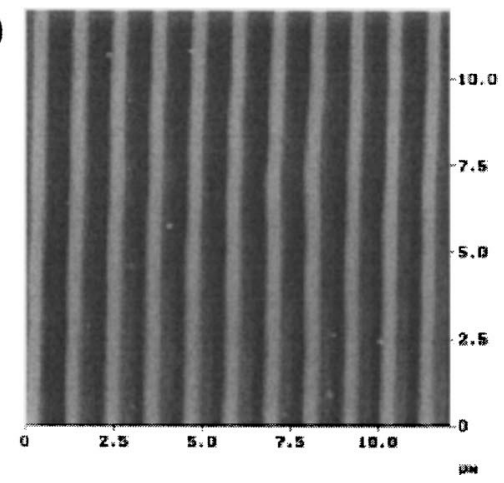

FIG. 1. AFM record of gratings $(\Lambda=1 \mu \mathrm{m})$ made by 1 pulse (a) on ITO at $305 \mathrm{~mJ} / \mathrm{cm}^{2}$ (b) on PI on ITO at $350 \mathrm{~mJ} / \mathrm{cm}^{2}$, and (c) on PMMA on ITO at $350 \mathrm{~mJ} / \mathrm{cm}^{2}(193 \mathrm{~nm}, 20 \mathrm{~ns})$.

polycrystalline $\mathrm{SiO}_{2}(20 \mathrm{~nm})$ layer on top of a $1.1 \mathrm{~mm}$ sodalime glass substrate. PI and PMMA were deposited on ITO coated glass substrates by spin coating with film thickness of 100 and $400 \mathrm{~nm}$, respectively. Grating structures of 700$625,330-416 \mathrm{~nm}$, and $1 \mu \mathrm{m}$ were prepared by a single laser shot. Different depth modulations were achieved by changing the laser fluence. At high fluences, the ITO layer can be structured down to the glass substrate, the depth modulation ranging between 24 and $3.6 \mathrm{~nm}$; for PI on ITO, the modulation varied between 25 and $0.5 \mathrm{~nm}$ and for PMMA on ITO it varied between 28 and $1.9 \mathrm{~nm}(193 \mathrm{~nm}, 20 \mathrm{~ns})$. The images of the ablated gratings were taken with an AFM in tapping mode. Figure 1 shows AFM images of surface gratings of about $25 \mathrm{~nm}$ depth made by one $20 \mathrm{~ns}$ pulse at $193 \mathrm{~nm}$ with $305 \mathrm{~mJ} / \mathrm{cm}^{2}$ on ITO (a), on PI coated ITO using one pulse at $350 \mathrm{~mJ} / \mathrm{cm}^{2}$ (b), and on PMMA coated ITO with one pulse at $350 \mathrm{~mJ} / \mathrm{cm}^{2}$ (c). Though all gratings are perfectly regular, the gratings in the polymer films are squarer shaped. Similar results were obtained using the fs pulses at $248 \mathrm{~nm}$ (not shown). The alignment of all the grating structures was investi- (a)

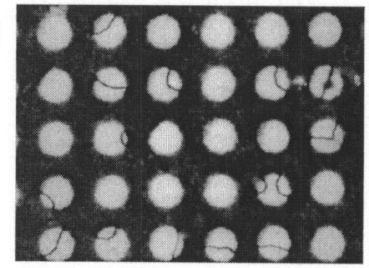

(b)

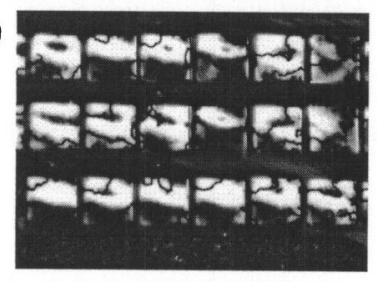

(c)

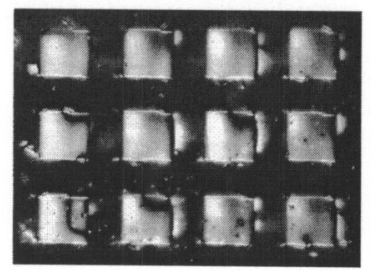

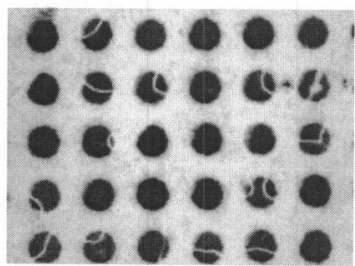
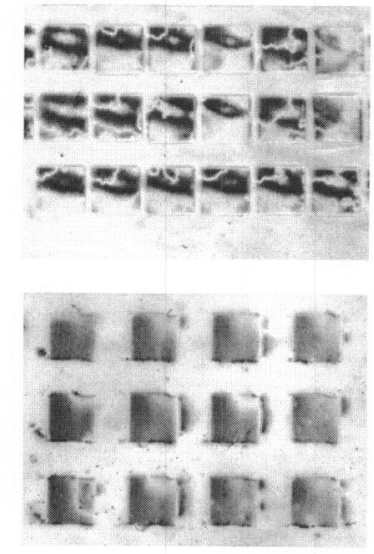

FIG. 2. TN pixels on (a) ITO under perpendicular (left) and parallel polarizer and analyzer (b) PI under perpendicular (left) and parallel polarizer and analyzer and (c) PMMA under perpendicular (left) and parallel polarizer and analyzer.

gated using twisted nematic (TN) cells filled with 5CB (Merck) at the isotropic phase $\left(40^{\circ} \mathrm{C}\right)$ by capillary action and, afterwards, slowly cooled down to room temperature $\left(23^{\circ} \mathrm{C}\right)$. Cells with a thickness of $6 \mu \mathrm{m}$ were prepared with alignment surfaces perpendicular to each other: one surface with the laser ablated grating and a conventionally rubbed PI counter plate.

Rubbed PI is known to strongly anchor the liquid crystal molecules in the direction of rubbing and therefore can be used as a reference surface. The direction of the grooves was oriented normal to the direction of the rubbed PI. The liquid crystal alignment was studied by polarized microscopy. The cell rotation $\operatorname{method}^{9}$ was used to calculate the surface anchoring energies, which was measured as function of the depth of the grating and width of the grooves using the following equation: ${ }^{10}$

$$
W_{\phi}=\frac{2 K_{22} \phi}{d \sin 2 \phi},
$$

where $K_{22}$ is the twist elastic constant and $d$ is the cell spacing. We found that for medium fluences $\left(125-140 \mathrm{~mJ} \mathrm{~cm}{ }^{2}\right)$ $W_{\phi}$ does neither depend on the depth nor on the width of the grooves for the investigated materials. The obtained anchoring energies are $6 \times 10^{-6} \mathrm{~J} \mathrm{~m}^{-2}$ for ITO, $1.6 \times 10^{-5} \mathrm{~J} \mathrm{~m}^{-2}$ for PI, and $1.05 \times 10^{-5} \mathrm{~J} \mathrm{~m}^{-2}$ for PMMA. The anchoring energies found for PI and PMMA are of the same order as for the conventionally rubbed PI, whereas the value for ITO is a factor of 3 smaller than that obtained by AFM patterning. ${ }^{6}$

Figure 2 shows optical micrographs of a series of TN pixels. LCs are well aligned in the grating area and form TN pixels. In Fig. 2 it can clearly be seen that LC molecules on the nonablated area are not aligned. In the case of ITO, there is no polymer chain alignment and for the polymers, we used nonpolarized UV light (though the attenuator could introduce to AIP license or copyright; see http://apl.aip.org/apl/copyright.jsp 
some polarization, as it consists of a turning dielectric plate). We can conclude that the alignment observed in these cells is due to the surface gratings.

Different LC domains can be clearly seen in most pixels of Figs. 2(a) and 2(b). Under crossed polarizers we observed different colors that show multiple twisted domains. Formation of these domains indicates that there is no pretilt angle on the patterned surfaces. ${ }^{5}$ The existence of a pretilt angle on traditionally rubbed polymer films is related to the stress applied to the polymer film by the rubbing. ${ }^{11}$ In absence of such unidirectional stress the LC molecules can form either a left- or a right-handed twist that energetically are degenerate. Note that these cells have been filled in the isotropic phase of the liquid crystal and therefore there is no flow alignment on the patterned surface to remove this degeneracy.

We observed that for high fluences $\left(>140 \mathrm{~mJ} \mathrm{~cm}^{-2}\right)$ and for low fluences $\left(110 \mathrm{~mJ} \mathrm{~cm}^{-2}\right)$, the gratings ablated on ITO and PI do not align the LC molecules very well. Gratings ablated on PMMA align the LC molecules for all fluences. Partly this can be understood: the alignment can be weak for too shallow gratings because they are not homogeneous whereas for the case of ITO at high fluences, we get down to the bare substrate $\left(\mathrm{SiO}_{2}\right)$ that tends to align molecules homeotropically and not planar. However, for PI at $25 \mathrm{~nm}$ maximum depth $\left(350 \mathrm{~mJ} \mathrm{~cm}^{-2}\right)$, there is still $75 \mathrm{~nm}$ of PI in contact with the LC molecules; we assume that the ablative photodecomposition process is responsible for this lack of alignment. After the absorption of a photon with higher energy than the molecular bond energy, deactivation processes like bond breaking (photodissociation) and vibrational relaxation take place. The latter leads to a heating of the surrounding material with possibility of decomposition via thermal activation. ${ }^{12}$ The resulting photoproducts and the modified ring structures of PI that remain on the surface can affect the LC-surface interaction. ${ }^{13}$ For PMMA, we noticed from the AFM observations that the quality of the grating is the best of the three materials investigated at $193 \mathrm{~nm}$. We also noticed that for fs pulses the alignment is good for all gratings.
In conclusion, we have shown that laser ablated period structures can be used to efficiently align liquid crystal molecules. The surface anchoring energy remains unchanged by changing either the depth or the width of the grooves for gratings of $1 \mu \mathrm{m}$ period. Saturated alignment is achieved by fluences above the threshold but good quality of alignment is only achieved by medium fluences. In this region (125-140 $\mathrm{mJ} \mathrm{cm}{ }^{-2}$ ), the gratings are homogeneous with the least photodegradation. Being above the ablation threshold, we can assume that the anchoring is due to the grating topology rather than a photoinduced polymer chain alignment.

S.S. acknowledges the European network Grant SILC, MAB the German Federal Department of Education and Research (BMBF), MaTech program ("Hot Topics"), and M. Behdani the Ministry of Research and Technology of Iran for his fellowship. This work was partly sponsored by the Dutch Technology Foundation STW.

${ }^{1}$ J. van Haaren, Nature (London) 411, 29 (2001).

${ }^{2}$ S. Faetti, Phys. Rev. A 36, 408 (1987); M. Barmentlo, N. A. J. M. van Aerle, R. W. J. Hollering, and J. P. M. Damen, J. Appl. Phys. 71, 4799 (1992).

${ }^{3}$ D. W. Berreman, Phys. Rev. Lett. 28, 1683 (1972).

${ }^{4}$ M. Schadt, H. Seiberle, and A. Schuster, Nature (London) 381, 212 (1996).

${ }^{5}$ A. Rastegar, M. Škarabot, B. Blij, and Th. Rasing, J. Appl. Phys. 89, 960 (2001).

${ }^{6}$ M. Behdani, A. Rastegar, S. H. Keshmiri, S. I. Missat, E. Vlieg, and Th. Rasing, Appl. Phys. Lett. 80, 4635 (2002).

${ }^{7}$ H. M. Phillips, D. L. Callahan, R. Sauerbrey, G. Szabo, and Z. Bor, Appl. Phys. A: Solids Surf. A54, 158 (1992).

${ }^{8}$ S. Szatmári and F. P. Schaefer, Opt. Commun. 68, 196 (1988).

${ }^{9}$ G. P. Bryan-Brown and I. C. Sage, Liq. Cryst. 20, 825 (1996).

${ }^{10}$ C. J. Newsome, M. O'Neill, R. J. Farley, and G. P. Bryan-Brown, Appl. Phys. Lett. 72, 2078 (1998).

${ }^{11}$ J. M. Geary, J. W. Goodby, A. R. Kmetz, and J. S. Patel, J. Appl. Phys. 62, 4100 (1987)

${ }^{12}$ H. Schmidt, J. Ihlemann, B. Wolf-Rottke, K. Luther, and J. Troe, J. Appl. Phys. 83, 5458 (1998).

${ }^{13}$ C. J. Newsome and M. O'Neill, J. Appl. Phys. 88, 7328 (2000). 